\title{
Advanced ovarian malignancy in pregnancy mimicking ovarian hyperstimulation syndrome: a case report
}

\author{
Mythili Kundur $^{1 *}$, Anupama Rajanbabu ${ }^{1}$, Vinita Murali ${ }^{2}$, Pavithran Keechilatt ${ }^{3}$
}

\author{
${ }^{1}$ Department of Gynecological Oncology, Amrita Institute of Medical Sciences, Kochi, Kerala, India \\ ${ }^{2}$ Department of Gynecology, Amrita Institute of Medical Sciences, Kochi, Kerala, India \\ ${ }^{3}$ Department of Medical Oncology, Amrita Institute of Medical Sciences, Kochi, Kerala, India
}

Received: 20 July 2021

Revised: 14 August 2021

Accepted: 16 August 2021

\author{
*Correspondence: \\ Dr. Mythili Kundur, \\ E-mail: kundru.mythili@gmail.com
}

Copyright: (C) the author(s), publisher and licensee Medip Academy. This is an open-access article distributed under the terms of the Creative Commons Attribution Non-Commercial License, which permits unrestricted non-commercial use, distribution, and reproduction in any medium, provided the original work is properly cited.

\begin{abstract}
Advanced ovarian malignancy is a rare occurrence in pregnancy. Here we report a case of primary infertility presenting in early pregnancy following invitro fertilization with features of Ovarian hyperstimulation syndrome unresponsive to treatment. Further evaluation revealed advanced ovarian malignancy. She was treated with chemotherapy followed by staging surgery at the time of elective cesarean at 35 weeks gestation. This case outlines the difficulties in diagnosis of ovarian cancer during pregnancy.
\end{abstract}

Keywords: Ovarian cancer, Epithelial ovarian cancer, OHSS, Diagnosis, IVF

\section{INTRODUCTION}

Ovarian cancer complicates 1 in 15,000 to 1 in 32,000 pregnancies, and is the second most common gynaecologic cancer diagnosed during pregnancy. ${ }^{1}$ A rising incidence during pregnancy in recent times could be attributable to increasing age of conception. ${ }^{1}$ However, advanced ovarian malignancy remains rare in pregnancy. The reported incidence of Ovarian hyperstimulation syndrome (OHSS) in Invitro fertilization (IVF) conceptions is estimated to be $3-6 \%$ for moderate and $0.1-2 \%$ for severe forms. ${ }^{2}$ Given the overlapping presentation with OHSS, differentiating advanced ovarian malignancy from this requires a high degree of suspicion.

Here we present a case of advanced ovarian malignancy that was presumptively diagnosed as OHSS.

\section{CASE REPORT}

A 39 years old primigravida at 12 weeks of gestation with gross abdominal distension was referred to the gynecology department of our hospital for management of severe OHSS. This pregnancy resulted after invitro fertilization (IVF). She gave a history of an unsuccessful invitro fertilization prior to the present pregnancy.

At presentation, she had grade 2 pedal edema, tense ascites and decreased breath sounds in the right infrascapular region.

Ultrasound imaging (USG) showed moderate ascites, bilateral pleural effusion and bilateral enlarged multicystic ovaries. The fetus was viable with normal growth interval.

Treatment was initiated for the OHSS. However, she showed no improvement and her ascites rapidly reaccumulated despite multiple paracentesis. Her metabolic parameters and beta hCG were normal.

Upon further investigation, MRI revealed bilateral bulky ovaries replaced by multiloculated cysts with solid areas and mural nodules, mild bilateral pleural effusion and moderate ascites. Carbohydrate antigen 125 (CA 125) was 
5756 units/ml. All other tumour markers were normal. She was referred to Gynaecological oncology department for evaluation as the imaging was suspicious for ovarian malignancy.

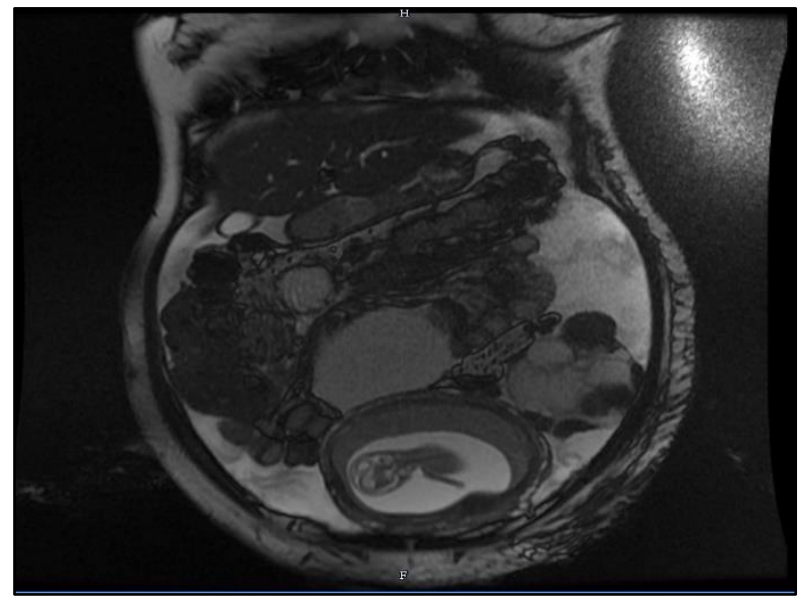

Figure 1: MRI 2D FIESTA coronal section showing bilateral multicytic ovarian masses with solid areas, ascites and a gravid uterus.

Evaluation at gynaecologic Oncology showed an enlarged left supraclavicular lymph node and fine needle aspiration cytology of the node revealed cells favouring adenocarcinoma. A diagnosis of carcinoma ovary stage IVB was made.

The case was discussed in a multidisciplinary tumour board. Pregnancy termination in view of advanced malignancy in early pregnancy was discussed with the patient and her husband but it was unacceptable to the them. Taking the patient's wishes into consideration, chemotherapy was started. She received 6 cycles of Paclitaxel and Carboplatin, the first dose at 14 weeks of gestation and the last at 31 weeks, with good clinical response.

She underwent elective caesarean section and debulking surgery for ovarian cancer at 35 weeks and 3 days of gestation. Optimal cytoreduction was achieved. A live female baby weighing $1.88 \mathrm{~kg}$ was delivered.

Histopathology revealed poorly differentiated carcinoma, suggestive of serous carcinoma involving both ovaries and the omentum.

Patient was disease free for 9 months following which she developed a peritoneal recurrence for which second line chemotherapy with Gemcitabine and carboplatin was given.

5 months later, she had a second recurrence with extensive peritoneal carcinomatosis. Further chemotherapy was given with Lipodox and carboplatin, but she succumbed to the disease 2 years after the initial diagnosis.

\section{DISCUSSION}

Some studies have reported a higher incidence of ovarian malignancy among infertile women. However, studies and systematic reviews show no association between the use of fertility treatments and invasive ovarian cancer compared to subfertile control groups. ${ }^{3}$

The diagnosis of ovarian malignancy in pregnancy is no less challenging than its management. Its symptoms are vague, more so in gravid women. This patient was mistakenly diagnosed as OHSS. This rare and misleading presentation has only been reported in two prior cases. ${ }^{4}$ Physiological symptoms associated with pregnancy including nausea, vomiting, abdominal discomfort, loss of appetite, shortness of breath and fatigue overlap with ovarian cancer and OHSS. Pain abdomen, Ascites, pleural effusion and enlarged ovaries raise a suspicion of OHSS in IVF pregnancies.

CA 125 as a tumour marker is elevated in $80-90 \%$ of epithelial ovarian tumors. It is unreliable in pregnancy and maybe elevated in upto $10 \%$ of normal gravid women, predominantly in the first trimester. ${ }^{5,6}$ In our case, CA 125 was markedly raised and offered the first clue to the diagnosis.

Laboratory parameters offer some insight only in more severe forms of OHSS with metabolic derangement, haemoconcentration and leucocytosis. All of these remained normal in this patient.

Routine ultrasound examination is integral to modern obstetrics and has led to increased detection of adnexal lesions that occur in $1-5.3 \%$ of pregnancies. ${ }^{7}$ Of these a mere $2-5 \%$ are malignant. ${ }^{8}$ A finding of bulky ovaries in the setting of an IVF pregnancy in this case mislead her to be diagnosed as OHSS. Presence of solid areas, persistence of the ovarian lesions beyond the first trimester, ascites not responsive to standard OHSS treatment should prompt further evaluation.

MRI is the optimal higher imaging to characterize these lesions in pregnancy as it avoids radiation exposure. The Assessment of Different Neoplasms in the Adnexa Magenetic resonance (ADNEX MR) scoring system showed $80 \%$ accuracy in cancer detection. ${ }^{1}$ In our case, it was only on imaging by MRI that the diagnosis of ovarian cancer was considered.

Reaching a conclusive diagnosis at the earliest is paramount for two reasons- surgical intervention where possible is ideal between 16-20 weeks of gestation and in advanced malignancy, termination of pregnancy is a reasonable option prior to 20 weeks. $^{9}$

\section{CONCLUSION}

Ovarian malignancy in pregnancy usually presents in early stage but can rarely present as advanced malignancy. The 
diagnosis is challenging owing to nonspecific symptoms. With routine ultrasound examination, adnexal masses are being increasingly detected in pregnancy. However due to the rarity of the condition, diagnosis of ovarian malignancy is often delayed. The possibility of ovarian cancer should be kept in mind while evaluating features of OHSS that are not responding to standard treatment.

Funding: No funding sources

Conflict of interest: None declared

Ethical approval: Not required

\section{REFERENCES}

1. Franciszek, Dłuski D, Mierzyński R, PoniedziałekCzajkowska E, Leszczyńska-Gorzelak B. Ovarian Cancer and Pregnancy-A Current Problem in Perinatal Medicine: A Comprehensive Review. Cancers (Basel). 2020;12(12):3795.

2. El Tokhy O, Kopeika J, El-Toukhy T. An update on the prevention of ovarian hyperstimulation syndrome. Womens Health (Lond). 2016;12(5):496-503.

3. Kroener L, Dumesic D, Al-Safi Z. Use of fertility medications and cancer risk: a review and update. Curr Opin Obstet Gynecol. 2017;29(4):195-201.
4. Ben-Hur H, Dgani R, Lancet M, Katz Z, Nissim F, Rosenman D. Ovarian carcinoma masquerading as ovarian hyperstimulation syndrome. Acta Obstet Gynecol Scand. 1986;65(7):813-4.

5. Amampai R, Suprasert P. Cancer Antigen 125 during Pregnancy in Women without Ovarian Tumor Is Not Often Rising. Obstet Gynecol Int. 2018;2018:8141583.

6. Han SN, Lotgerink A, Gziri MM. Physiologic variations of serum tumor markers in gynecological malignancies during pregnancy: a systematic review. BMC Med. 2012;10:86.

7. Martone S, Troìa L, Luisi S. Adnexal masses during pregnancy: management for a better approach. Gynecol Surg. 2021;18:3.

8. T RK, Korenaga KS. Tewari, Gynecologic cancer in pregnancy, Gynecologic Oncology. 2020;127(3):799809.

9. Amant F, Berveiller P, Boere IA, Cardonic E. Gynecologic cancers in pregnancy: Guidelines based on a third international consensus meeting, Annals of Oncology. 2019;30(3):1601-12.

Cite this article as: Kundur M, Rajanbabu A, Murali V, Keechilatt P. Advanced ovarian malignancy in pregnancy mimicking ovarian hyperstimulation syndrome: a case report. Int J Reprod Contracept Obstet Gynecol 2021;10:3647-9. 